\title{
Liste des tables
}

1.1. Unités de base du Système international d'unités (SI) . . . . . . . . . . 14

1.2. Définition des sept unités de base du SI . . . . . . . . . . . . . . . 15

1.3. Unités usuelles en dehors du SI . . . . . . . . . . . . . . . . . 17

1.4. Unités de base du système UAI d'unités astronomiques . . . . . . . . . 19

1.5. Correspondance entre les unités de temps . . . . . . . . . . . . . 19

1.6. Correspondance entre les unités de distance . . . . . . . . . . . . 20

1.7. Le système UAI 2009/2012 de constantes astronomiques . . . . . . . . . . . . 25

1.8. Valeurs de quelques constantes dérivées . . . . . . . . . . . . 28

1.9. Valeurs numériques IERS 2010 de constantes relatives à la Terre $\ldots . .28$

1.10. Système UAI 2015 des constantes de conversion nominales solaires et planétaires ........................ 30

1.11. Rapport de la masse du Soleil aux masses des planètes et planètes naines 31

1.12. Rayons équatoriaux des planètes, de la Lune et du Soleil . . . . . . . . . 31

1.13. Champ de gravitation des planètes . . . . . . . . . . . . . 32

1.14. Champ de gravitation de la Lune . . . . . . . . . . . . . . 33

1.15. Éléments orbitaux des planètes rapportés à l'écliptique et à l'équinoxe

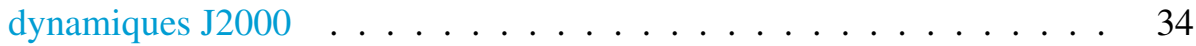

1.16. Périodes de révolution sidérale et de rotation des planètes . . . . . . . . 35

1.17. Éléments orbitaux des satellites de planètes et planètes naines découverts

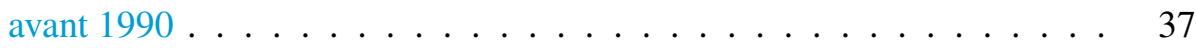

1.18. Éléments orbitaux des satellites découverts après 1990 . . . . . . . . . 40

1.19. Masses des corps célestes et constantes gravitationnelles correspondantes 46

1.20. Figures géométriques représentant le Soleil, les planètes et la Lune . . . 47

1.21. Masses des principaux satellites naturels . . . . . . . . . . . . . . 49

1.22. Masse, masse volumique, magnitude visuelle à l'opposition et albédo géométrique des principaux satellites . . . . . . . . . . 51

2.1. Brève histoire des échelles de temps modernes . . . . . . . . . . . 60

2.2. Différence TAI - UTC, en secondes, à partir du $1^{\mathrm{er}}$ janvier $1972 \ldots . . .71$

2.3. Amplitudes, fréquences et phases donnant la différence $P=$ TDB - TT . $\quad 80$

2.4. Correspondance entre époques, dates juliennes et dates calendaires. . . . 88

2.5. Différences TT - UT, en secondes, de -1000 à 1550 . . . . . . . . . 92

2.6. Différences TT - UT, en secondes, de 1600 à 2015 . . . . . . . . . . . . 94 


\section{LISTE DES TABLES}

3.1. Observations utilisées dans DE405 pour effectuer le lien avec l'ICRF . . 123

3.2. Angles de position de l'écliptique moyen J2000.0 par rapport à un système céleste équatorial . . . . . . . . . . . . . . . . . 125

3.3. Termes périodiques les plus importants des coordonnées $X(t)$ et $Y(t)$ du CIP dans le GCRS

4.1. Expressions des coefficients en $t$ et $t^{2}$ des quantités de précession . . . .

4.2. Coefficients des polynômes de la précession pour les modèles UAI 1976, UAI 2000 et UAI $2006 \ldots \ldots \ldots$

5.1. Sources et argument des éphémérides planétaires de la Connaissance des temps depuis $1809 \ldots \ldots$. . . . . . . . . . . . . . .

5.2. Éléments elliptiques et masse de Neptune comparés aux valeurs prévues par Le Verrier et Adams . . . . . . . . . . . . . . . . . . . . 225

5.3. Rapport de la masse du Soleil à la masse des systèmes planétaires . . . . 239

5.4. GM des corps entrant dans la construction de INPOP10a . . . . . . . . . 246

5.5. Différences maximales sur 1890 - 2000 entre VSOP2013, VSOP2010, TOP2013 et les intégrations numériques . . . . . . . . . . . .

5.6. Différences maximales sur de longues durées entre VSOP2013, TOP2013 et l'extension de INPOP10a . . . . . . . . . . . . . . . .

5.7. Termes trigonométriques complémentaires pour les éléments moyens des planètes . . . . . . . . . . . . . . . . . . . 272

5.8. Erreurs maximales des éphémérides approchées des planètes . . . . . . 276

5.9. Principaux termes périodiques du demi-grand axe et de la longitude moyenne de la Lune . . . . . . . . . . . . . . . . . . . 282

5.10. Principaux termes périodiques dans l'excentricité et la longitude du périgée de l'orbite lunaire . . . . . . . . . . . . . . . . .

5.11. Principaux termes périodiques sur l'inclinaison et la longitude du nœud de l'orbite lunaire . . . . . . . . . . . . . . . . . . . . . . 289

5.12. Composantes d'amplitude $>10^{\prime \prime}$ dans la longitude de la Lune . . . . . 295

5.13. Composantes d'amplitude $>10^{\prime \prime}$ dans la latitude de la Lune . . . . . . 296

5.14. Composantes d'amplitude $>20 \mathrm{~km}$ dans la distance de la Lune . . . . . 297

5.15. Variation temporelle des fréquences pour les plus grandes perturbations de l'orbite lunaire . . . . . . . . . . . . . . . . . . . . . 298

5.16. Liste des plus petits périgées lunaires . . . . . . . . . . . . 303

5.17. Paramètres ajustés pour différentes éphémérides . . . . . . . . . . . . . 304

5.18. Différences maximales en positions entre les versions d'INPOP . . . . 308

5.19. Différences maximales pour la Terre entre les versions d'INPOP . . . . 308

5.20. Valeurs de paramètres fondamentaux pour diverses versions de INPOP 309

5.21. Données utilisées pour la construction des solutions INPOP . . . . . . 312

5.22. Intervalles de valeurs pour des paramètres ajustés avec INPOP . . . . . 314

5.23. Paramètres de forme pour la Terre la Lune ajustés avec INPOP17a . . . 317 


\section{LISTE DES TABLES}

5.24. Statistiques des résidus obtenus après ajustement d'INPOP17a . . . . . 318

6.1. Ecarts au mouvement uniforme . . . . . . . . . . . . . . . . . . . . 324

6.2. Nombre de satellites naturels connus . . . . . . . . . . . . . . . . . . 328

6.3. Observations disponibles des satellites de Jupiter et Saturne . . . . . . . 346

6.4. Observations disponibles des satellites d'Uranus, Neptune et Pluton) . . 347

6.5. Nombre de phénomènes observés . . . . . . . . . . . . . . . . . 347

6.6. Précision astrométrique interne des observations . . . . . . . . . . . . . . . 349

6.7. Termes principaux de la longitude moyenne de Io . . . . . . . . . . 356

6.8. Termes principaux de la longitude moyenne de Mimas . . . . . . . . . . 357

6.9. Valeurs approchées d'éléments des orbites de Phobos et Déimos . . . . . 366

6.10. Éléments moyens de Struve rapportés à J2000 . . . . . . . . . . . . 372

6.11. Valeurs des termes périodiques des éléments de Struve . . . . . . . . 373

6.12. Éphémérides des satellites galiléens dans la Connaissance des temps . . 377

6.13. Eléments orbitaux et paramètres physiques des satellites galiléens . . . 378

6.14. Différentes valeurs des accélérations séculaires des satellites galiléens . 380

6.15. Rayons des anneaux de Jupiter . . . . . . . . . . . . . . . . . . . 388

6.16. Éléments des satellites internes de Jupiter . . . . . . . . . . . . . . 388

6.17. Déviations des éphémérides JPL et TSU . . . . . . . . . . . . . . . 391

6.18. Paramètres de l'ellipse précessante pour les satellites Métis et Adrastée 392

6.19. Paramètres de l'ellipse précessante pour les satellites Amalthée et Thébé 393

6.20. Liste des satellites de Saturne . . . . . . . . . . . . . . . . 395

6.21. Caractéristiques dynamiques des neuf premiers satellites de Saturne . . 397

6.22. Éléments orbitaux initiaux de Mimas, Encelade, Téthys et Dioné . . . . 401

6.23. Paramètres associés aux librations affectant les couples Mimas-Téthys et Encelade-Dioné . . . . . . . . . . . . . . . . . . . . 401

6.24. Éléments orbitaux initiaux de Rhéa . . . . . . . . . . . . . . 403

6.25. Éléments orbitaux initiaux de Titan . . . . . . . . . . . . . . 404

6.26. Éléments orbitaux initiaux de Japet . . . . . . . . . . . . . . . . . 406

6.27. Éléments orbitaux initiaux d'Hypérion . . . . . . . . . . . . . . . . 409

6.28. Conditions initiales de l'orbite de Phøébé . . . . . . . . . . . . . 410

6.29. Principales caractéristiques orbitales des satellites proches de Saturne . 412

6.30. Solution pour la variable $p_{1}$ (moyen mouvement de Mimas) . . . . . . 419

6.31. Solution pour la variable $\lambda_{1}$ (longitude moyenne de Mimas). . . . . . . 420

6.32. Solution pour la variable $z_{1}$ (excentricité et péricentre de Mimas). . . . 420

6.33. Solution pour la variable $\zeta_{1}$ (inclinaison et nœud de Mimas) . . . . . . 421

6.34. Solution pour la variable $p_{2}$ (moyen mouvement d'Encelade) . . . . . . 421

6.35. Solution pour la variable $\lambda_{2}$ (longitude moyenne d'Encelade). . . . . . 421

6.36. Solution pour la variable $z_{2}$ (excentricité et péricentre d'Encelade) . . . 421

6.37. Solution pour la variable $\zeta_{2}$ (inclinaison et nœud d'Encelade) . . . . . . . 422

6.38. Solution pour la variable $p_{3}$ (moyen mouvement de Téthys) . . . . . . 422

6.39. Solution pour la variable $\lambda_{3}$ (longitude moyenne de Téthys) . . . . . . 422 


\section{LISTE DES TABLES}

6.40. Solution pour la variable $z_{3}$ (excentricité et péricentre de Téthys) . . . . 422

6.41. Solution pour la variable $\zeta_{3}$ (inclinaison et nœud de Téthys) . . . . . . . 423

6.42. Solution pour la variable $p_{4}$ (moyen mouvement de Dioné) . . . . . . . 423

6.43. Solution pour la variable $\lambda_{4}$ (longitude moyenne de Dioné) . . . . . . . 423

6.44. Solution pour la variable $z_{4}$ (excentricité et péricentre de Dioné) . . . . 423

6.45. Solution pour la variable $\zeta_{4}$ (inclinaison et nœud de Dioné) . . . . . . . . . 424

6.46. Solution pour la variable $p_{5}$ (moyen mouvement de Rhéa) . . . . . . . 424

6.47. Solution pour la variable $\lambda_{5}$ (longitude moyenne de Rhéa) . . . . . . . 424

6.48. Solution pour la variable $z_{5}$ (excentricité et péricentre de Rhéa) . . . . . 425

6.49. Solution pour la variable $\zeta_{5}$ (inclinaison et nœud de Rhéa) . . . . . . . 425

6.50. Solution pour la variable $p_{6}$ (moyen mouvement de Titan) . . . . . . . 425

6.51. Solution pour la variable $\lambda_{6}$ (longitude moyenne de Titan) . . . . . . 426

6.52. Solution pour la variable $z_{6}$ (excentricité et péricentre de Titan) . . . . . 426

6.53. Solution pour la variable $\zeta_{6}$ (inclinaison et nœud de Titan) $\ldots . .4 .426$

6.54. Solution pour la variable $p_{8}$ (moyen mouvement de Japet) $\ldots . . .427$

6.55. Solution pour la variable $\lambda_{8}$ (longitude moyenne de Japet) . . . . . . . 427

6.56. Solution pour la variable $z_{8}$ (excentricité et péricentre de Japet) . . . . . 429

6.57. Solution pour la variable $\zeta_{8}$ (inclinaison et nœud de Japet) . . . . . . . 430

6.58. Arguments fondamentaux de l'interaction Titan-Hypérion . . . . . . . 431

6.59. Plus grands termes de la série pour $p_{7} \ldots \ldots$. . . . . . . . . . 432

6.60. Plus grands termes de la série pour $q_{7} \ldots \ldots$. . . . . . . . . . . . . 432

6.61. Plus grands termes de la série pour $z_{7}=e_{7} \exp \sqrt{-1} \varpi_{7} \ldots \ldots$. . . . 434

6.62. Plus grands termes de la série pour $\zeta_{7}=\sin \left(i_{7} / 2\right) \exp \sqrt{-1} \Omega_{7} \ldots \ldots 435$

6.63. Perturbations solaires et à courte période d'Hypérion . . . . . . . . . . . 436

6.64. Éléments orbitaux des satellites intérieurs d'Uranus . . . . . . . . . . . . . 442

6.65. Eléments orbitaux des cinq satellites principaux d'Uranus . . . . . . . 443

6.66. Masses et fréquences des cinq satellites principaux d'Uranus . . . . . . 447

6.67. Paramètres du système de Neptune . . . . . . . . . . . . . . . . . . . . . 454

6.68. Éléments orbitaux planétocentriques moyens de Triton . . . . . . . . . 455

6.69. Éléments orbitaux barycentriques moyens de Néréide . . . . . . . . . 455

6.70. Éléments orbitaux planétocentriques moyens de Protée, Larissa et Despina457

6.71. Éléments orbitaux planétocentriques moyens de Galatée, Thalassa et Naïade . . . . . . . . . . . . . . . . . . . . 458

6.72. Caractéristiques et éléments orbitaux des satellites de Pluton . . . . . . . 460

6.73. Éléments orbitaux des satellites directs irréguliers de Jupiter . . . . . . 463

6.74. Éléments orbitaux des satellites rétrogrades irréguliers de Jupiter . . . . 463

6.75. Éléments orbitaux des satellites directs irréguliers de Saturne . . . . . . 465

6.76. Éléments orbitaux des satellites rétrogrades irréguliers de Saturne . . . 465

6.77. Éléments orbitaux des satellites irréguliers d'Uranus . . . . . . . . . . . . 467

6.78. Éléments orbitaux des satellites irréguliers de Neptune . . . . . . . . . 467

6.79. Précision et exactitude des éphémérides des satellites naturels . . . . . 472 


\section{LISTE DES TABLES}

7.1. Évolution du calcul du passage au périhélie de la comète de Halley . . 496

7.2. Définition des sous-catégories des comètes . . . . . . . . . . . . . 503

7.3. Définition des sous-catégories des petits corps autres que les comètes . . 504

7.4. Troncatures des éléments osculateurs dans les bases ASTORB et MPCORB516

7.5. Statistiques sur les écarts des conditions initiales de la base ASTORB . . 517

7.6. Statistique globale des résidus d'ajustement . . . . . . . . . . . 518

7.7. Caractéristiques dynamiques du système (22) Kalliope . . . . . . . . . . 534

7.8. Caractéristiques dynamiques du système (31) Euphrosyne . . . . . . . . 535

7.9. Caractéristiques dynamiques du système (41) Daphne . . . . . . . . . 535

7.10. Caractéristiques dynamiques du système (45) Eugenia . . . . . . . . . . . 536

7.11. Caractéristiques dynamiques du système (87) Sylvia . . . . . . . . . . . 537

7.12. Caractéristiques dynamiques du système (93) Minerva . . . . . . . . . . . 538

7.13. Caractéristiques dynamiques du système (107) Camilla . . . . . . . . . 539

7.14. Caractéristiques dynamiques du système (130) Elektra . . . . . . . . . 540

7.15. Caractéristiques dynamiques du système (283) Emma . . . . . . . . . 540

7.16. Caractéristiques dynamiques du système (379) Huenna . . . . . . . . . 541

7.17. Caractéristiques dynamiques du système (617) Patroclus . . . . . . . . 541

7.18. Caractéristiques dynamiques du système (624) Hektor . . . . . . . . . 542

7.19. Caractéristiques dynamiques du système (702) Alauda . . . . . . . . . . 542

7.20. Caractéristiques dynamiques du système (762) Pulcova . . . . . . . . . . . 543

7.21. Caractéristiques dynamiques du système (50000) Quaoar . . . . . . . . 543

7.22. Caractéristiques dynamiques du système (90482) Orcus . . . . . . . . 544

7.23. Caractéristiques dynamiques du système (136108) Haumea . . . . . . . 545

7.24. Caractéristiques dynamiques du système (174567) Varda . . . . . . . . 546

8.1. Orbites nominales des systèmes GNSS ． . . . . . . . . . . . . . 563

8.2. Coordonnées des 5 points de Lagrange . . . . . . . . . . . . . . . 567

8.3. Distance des deux corps primaires aux points de Lagrange . . . . . . . . 568

8.4. Sondes envoyées aux points de Lagrange du Système Soleil-Terre . . . . 570

8.5. Description des « Deux lignes NORAD . . . . . . . . . . . . . . 591

9.1. Formules numériques approximatives pour la réfraction $\ldots \ldots$. . . . 635

9.2. Réfraction normale pour $z_{0}$ de $80^{\circ}$ à $90^{\circ} \ldots \ldots \ldots$. . . . . . . . . 636

9.3. Paramètres de définition de l'atmosphère standard . . . . . . . . . . . . 641

10.1. Moyens mouvements du Soleil et de la Lune . . . . . . . . . . . . . . 648

10.2. Liste des plus courtes et plus longues lunaisons . . . . . . . . . . 655

10.3. Dépression de l'horizon et réfraction dans l'horizon en fonction de l'altitude 672

10.4. Liste des dix plus favorables oppositions périhéliques de Mars . . . . . 690

10.5. Conditions générales pour les passages des planètes inférieures sur le disque solaire . . . . . . . . . . . . . . 700 


\section{LISTE DES TABLES}

10.6. Variations séculaires des éléments des orbites de Mercure, Vénus et de la Terre ......................... 701

10.7. Périodes de retour aux nœuds pour les passages de Mercure et de Vénus 704

10.8. Retours des passages de Mercure . . . . . . . . . . . . . . . 704

10.9. Retours des passages de Vénus . . . . . . . . . . . . . . . . . 706

10.10. Passages de Mercure sur le disque solaire de 1950 à 2050 . . . . . . . 709

10.11. Passages de Vénus sur le disque solaire de 1631 à 2255 . . . . . . . . 711

10.12. Passages sur le Soleil vus de Mars ou de Saturne . . . . . . . . . . . . 713

10.13. Phénomènes mutuels des satellites galiléens . . . . . . . . . . . . . 741

10.14. Liste de phénomènes mutuels des satellites de Saturne et d'Uranus . 742

11.1. Valeurs extrêmes et moyennes des paramètres d'une éclipse de Soleil . 769

11.2. Valeurs extrêmes et moyennes des paramètres d'une éclipse de Lune . . 814

12.1. Définition des systèmes de rotation du Soleil et des planètes . . . . . . . 842

12.2. Définition des systèmes de rotation des satellites . . . . . . . . . . . . 845

12.3. Définition des systèmes de rotation des astéroïdes . . . . . . . . . . . . . . . 852

12.4. Paramètres de forme des planètes . . . . . . . . . . . . . . . 855

12.5. Paramètres de forme des satellites . . . . . . . . . . . . . . . . 856

12.6. Paramètres de forme des astéroïdes . . . . . . . . . . . . . . . . 860

12.7. Paramètres de forme des comètes . . . . . . . . . . . . . . . . . . . 861

13.1. Durées des années équinoxiales et solsticiales de 2020 à 2040 . . . . . 866

13.2. Approximation calendaire de l'année lunaire . . . . . . . . . . . . 875

13.3. Approximation calendaire de l'année tropique moyenne . . . . . . . 876

13.4. Dates de début des saisons de 2010 à 2050 . . . . . . . . . . . . 887

13.5. Calendrier perpétuel lunaire du comput dionysien . . . . . . . . . . . 892

13.6. Dates des pleines lunes pascales postérieures ou égales au 21 mars . . . 893

13.7. Lettres dominicales au voisinage du bissexte . . . . . . . . . . . . . 893

13.8. Calendrier limité à la période du 21 mars au 25 avril . . . . . . . . . . 894

13.9. Correspondance entre le nombre d'or et les épactes juliennes . . . . . . . . 897

13.10. Calendrier lunaire perpétuel du comput grégorien . . . . . . . . . . . 900

13.11. Liste des sauts d'épactes jusqu'en 4500 . . . . . . . . . . . . . . . . . 901

13.12. Épactes grégoriennes de 1582 à 4499 . . . . . . . . . . . . . . . . . . 903

13.13. Comparaison nouvelles lunes pascales et nouvelles lunes vraies . . . . 904

13.14. Calendrier limité à la période du 21 mars au 25 avril . . . . . . . . . . . . . 904

13.15. Algorithme du calcul de la date de Pâques . . . . . . . . . . . . . . 906

13.16. Formulaire pour le calcul de la date de Pâques . . . . . . . . . . . . . 909

A.1. Coordonnées célestes usuelles . . . . . . . . . . . . . . . . . 921 